\title{
Efficient Synthesis of 2,3-Disubstituted Benzo[b]thiophenes Starting from 2-Aminobenzophenone
}

\author{
Kwanghee Koh Park" and Ju Hee Lee \\ Department of Chemistry, Chungnam National University, Daejeon 305-764, Korea. "E-mail: khkoh@cmuac.kr \\ Received September 14, 2008
}

Key Words : 2-Thiocyanatobenzophenone, 2-Mercaptobenzophenone, 2-Alkylmercaptobenzophenones, Benzo[b]thiophenes

Benzo $[b]$ thiophene derivatives have been reported to have many interesting biologically properties ${ }^{1-6}$ and the synthesis and derivatization of the benzo $[b]$ thiophene ring have drawn much interests. ${ }^{1-11}$ The syntheses of the benzo[b]thiophene ring were usually accomplished by the formation of a fused thiophene ring starting from appropriate benzene derivatives through the formation of one bond ' $c$ ', ' $b$ ', ' $c$ ', or ' $d$ ', or the formation of two bonds ' $a$ ' and ' $b$ ', ' $a$ ' and ' $c$ ', or ' $b$ ' and ' $c$, $1-6.8-11$ The fused thiophene ring synthesis through the formation of ' $b$ ' and ' $c$ ' bonds would readily provide 2,3 disubstituted benzo $[b]$ thiophene derivatives. For the thiophene ring synthesis through that two-bond formation, 2mercaptobenzoyl derivatives 1 or their precursor compounds are necessary as starting materials. However, only a few 2mercaptobenzoyl derivatives or their precursors are readily available. $\$ 1213$<smiles>c1ccc2[se]ccc2c1</smiles>

benzo $[b]$ thiophene ring<smiles>[Y]C(=O)c1ccccc1S</smiles>

1
Here, we report an improved synthesis of 2-thiocyanatobenzophenone 2 , which can be served as a precursor of 2mercaptobenzophenone, from 2-aminobenzophenone and the direct one-pot synthesis of 2,3-disubstituted benzo[b]thiophenes from 2 .

2-Thiocyanatobenzophenone 2 was prepared from 2aminobenzophenone by diazotization followed by Sandmeyer-type reaction. ${ }^{12 j 4}$ The reported procedure for the synthesis of 2 consists of diazotization of 2-aminobenzophenone, the isolation of the diazonjum compound as the fluoroborate salt, and then treatment with $\mathrm{CuSCN} / \mathrm{KSCN}$ to give 2 in $18 \%$ yield. ${ }^{12}$ We were able to improve the yield to $58 \%$ via a one-pot reaction from 2-aminobenzophenone without isolation of the diazonjum salt (Scheme 1).
Hydrolysis of the compound 2 was attempted using $\mathrm{NaOH}$ or $\mathrm{KOH}$ as a base to obtain the corresponding thiophenol $\mathbf{3}$, but the yield of 3 was very low and variable: the major isolated product was the disulfide 4 , resulting from the oxidation of the thiophenol 3 , even though much effort was exercised to exclude oxygen (Scheme 2). ${ }^{13.15}$ Reduction of 4 with triphenylphosphine didn't give a satisfactory yield of 3 either ${ }^{16}$ : tlc indicated that the reaction proceeded smoothly, but 3 appears to be re-oxidized to 4 during work-up process. Thus, we decided to carry out the hydrolysis and then alkylation reaction in a one-pot, without attempting the isolation of the hydrolyzed product, 3 .

A hydrolysis reaction mixture obtained from stirring a solution of the thiocyanatobenzophenone 2 and $\mathrm{NaOH}$ in $\mathrm{DMF} /$ water was treated with alkyl bromide $\left(\mathrm{BrCH}_{2} \mathrm{Y}: \mathrm{Y}=\right.$ $\mathrm{CN}, \mathrm{CO}_{2} \mathrm{Et}$, $\mathrm{COPh}$, COPh-4-OMe, or COPh-4-Me), stirred at $35{ }^{\circ} \mathrm{C}$ for $3-5 \mathrm{~h}$ and then quenched with $10 \%$ aq $\mathrm{HCl}$. Work-up of the reaction mixture gave the corresponding benzo[ $b]$ thiophene derivatives 7 . The $S$-alkylated product 5 could not be isolated, implying that four steps to give 7 from 2 , hydrolysis, $S$-alkylation, cyclization, and then dehydration, occur consecutively in one-pot (Scheme 2). The yields of 7 were in the range of $50-80 \%$ based on the starting material 2. Considering that four steps are involved in the transformation of 2 to 7 , it is certain that the reactions are quite efficient and convenient. 2,3-Disubstituted benzo[b]thiophenes 7a-e synthesized in this study are either new (7e) or have previously been prepared individually by a specific and/or complicated method from not-readily available starting materials. ${ }^{617-19}$

In conclusion, 2-thiocyanatobenzophenone 2 was easily prepared in moderate yield from readily available 2-aminobenzophenone via diazotization followed by reaction with $\mathrm{CuSCN} / \mathrm{KSCN}$. Treatment of 2 with $\mathrm{NaOH}$ and then alkyl halide in DMF-water provided 2,3-disubstituted benzo[b]thiophenes 7a-e in good yields: hydrolysis of 2 to thiol,<smiles>CCCC(=O)c1ccccc1N</smiles>

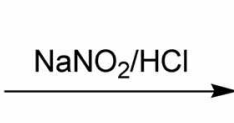<smiles>C#[N+]c1ccccc1C(=O)c1ccccc1</smiles>

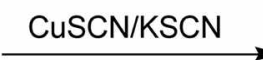<smiles>N#Cc1ccccc1C(=O)c1ccccc1</smiles>

$2(58 \%)$ 


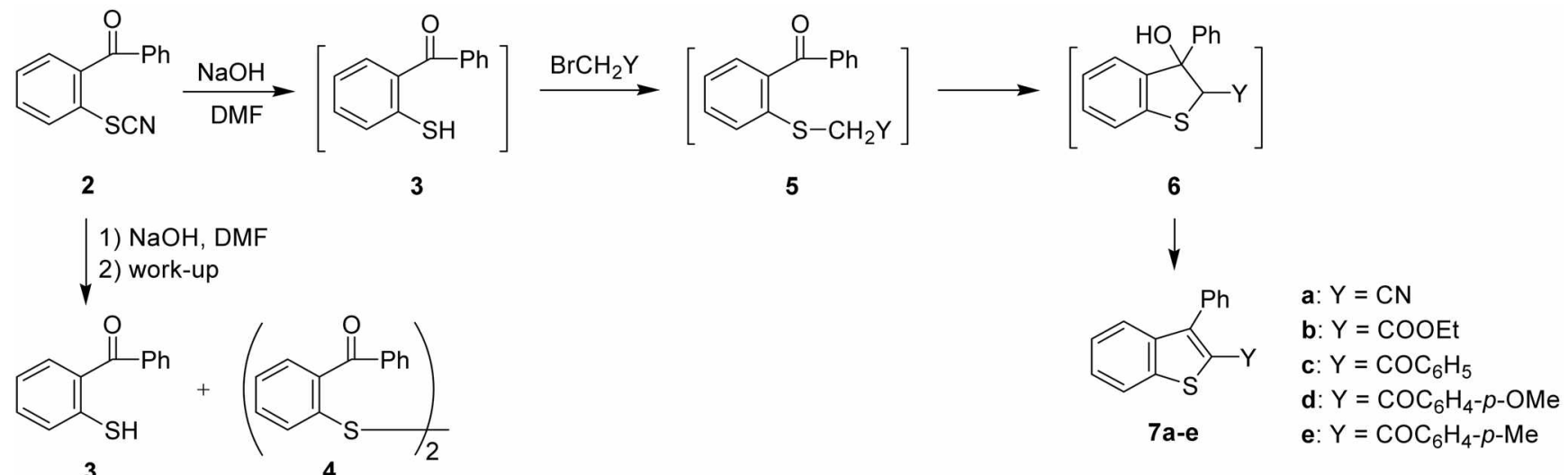

Scheme 2. Synthesis of 2,3-disubstituted benzo[ $b]$ thiophenes starting from 2-thiocyanatobenzophenone.

alkylation of the thiol, and then ring-closure/dehydration through Aldol-type condensation reaction occur consecutively in one-pot. We believe that our synthetic method described here provides a general, cheap, and efficient route for the synthesis of 2,3-disubstituted benzo[b]thiophenes.

\section{Experimental Section}

Melting points are uncorrected. ${ }^{1} \mathrm{H}$ and ${ }^{13} \mathrm{C}$ NMR spectra were obtained at 400 and $100 \mathrm{MHz}$, respectively, using tetramethylsilane (in $\mathrm{CDCl}_{3}$ ) as an internal standard.

Preparation of 2-thiocyanatobenzophenone 2 . The literature procedure 12,14 $^{2}$ was followed with slight modification. A solution of sodium nitrite $(0.77 \mathrm{~g}, 11 \mathrm{mmol})$ in water $(2 \mathrm{~mL})$ was added slowly over $15 \mathrm{~min}$ to a mixture of 2-aminobenzophenone $(2.00 \mathrm{~g}, 10.1 \mathrm{mmol})$, conc. $\mathrm{HCl}(3.0 \mathrm{~mL})$, and water $(30.0 \mathrm{~mL})$ at $0^{\circ} \mathrm{C}$ and the reaction mixture was stirred for additional $20 \mathrm{~min}$ at $0^{\circ} \mathrm{C}$ under $\mathrm{N}_{2}$ atmosphere. This reaction mixture was added slowly to a solution of CuSCN $(2.0 \mathrm{~g}, 16 \mathrm{mmol})$ and $\mathrm{KSCN}(19.5 \mathrm{~g}, 0.201 \mathrm{~mol})$ in water (20 $\mathrm{mL}$ ) at $0{ }^{\circ} \mathrm{C}$ and then the mixture was stirred at room temperature for $1 \mathrm{~h}$ and then at $60^{\circ} \mathrm{C}$ for 30 min under $\mathrm{N}_{2}$ atmosphere. The mixture was cooled to room temperature and the solid was collected by filtration. The solid was dried, and then extracted three times with boiling petroleum ether $(3 \times 40 \mathrm{~mL})$. The extracts were concentrated and the residue was purified by silica gel column chromatography eluting with $20: 1$ hexane-ethyl acetate to provide $1.39 \mathrm{~g}(58 \%)$ of 2thiocyanatobenzophenone $2: \mathrm{mp} 83^{\circ} \mathrm{C}$ (lit. ${ }^{12} 82-82.5^{\circ} \mathrm{C}$ ); ${ }^{1} \mathrm{H}$ $\operatorname{NMR}\left(\mathrm{CDCl}_{3}\right) \delta 8.02(\mathrm{~d}, 1 \mathrm{H}, J=8.0 \mathrm{~Hz}), 7.78-7.73(\mathrm{~m}, 3 \mathrm{H})$, $7.69-7.62(\mathrm{~m}, 2 \mathrm{H}), 7.52(\mathrm{t}, 2 \mathrm{H}, J=7.6 \mathrm{~Hz}), 7.43(\mathrm{t}, \mathrm{lH}, J=$ $7.6 \mathrm{~Hz}$ ); IR (KBr) 2154, 1635, 1586, 1438, 1322, 1313, $1273,737,730,696 \mathrm{~cm}^{-1}$.

Preparation of 2,3-disubstituted benzo[b]thiophenes 7a-e. A solution of 2-thiocyanatobenzophenone $2(0.20 \mathrm{~g}$, $0.84 \mathrm{mmol})$ in DMF ( $3 \mathrm{~mL}$ ) was added slowly to a solution of $\mathrm{NaOH}(0.334 \mathrm{~g}, 8.4 \mathrm{mmol})$ in water $(3 \mathrm{~mL})$ under $\mathrm{N}_{2}$ atmosphere and the reaction mixture was stirred at $35^{\circ} \mathrm{C}$ for 3 h. To this mixture was added slowly alkyl bromide $(1.7$ mmol) $\mathrm{BrCH}_{2} \mathrm{Y}$ (Y = CN, $\mathrm{CO}_{2} \mathrm{Et}, \mathrm{COPh}, \mathrm{COPh}-4-\mathrm{OMe}$, or COPh-4-Me) dissolved in DMF $(2 \mathrm{~mL})$. The reaction mix- ture was stirred at $35^{\circ} \mathrm{C}$ for $3-5 \mathrm{~h}$ and then quenched by neutralization with $10 \%$ aq $\mathrm{HCl}$. Saturated aq $\mathrm{NH}_{4} \mathrm{Cl}$ solution $(15 \mathrm{~mL})$ was added to the mixture and extracted with $\mathrm{CH}_{2} \mathrm{Cl}_{2}(3 \times 20 \mathrm{~mL})$. The organic layers were combined, dried with anhydrous $\mathrm{MgSO}_{4}$, concentrated, and then purified by silica gel column chromatography eluting with $20: 1$ hexane-ethyl acetate to provide the corresponding $2,3-$ disubstituted benzo[ $b]$ thiophene 7 .

2-Cyano-3-phenylbenzo[b]thiophene 7a: yield $59 \%$; mp $88^{\circ} \mathrm{C}$ (lit. ${ }^{17} 78-81^{\circ} \mathrm{C}$ ); ${ }^{1} \mathrm{H}$ NMR $\left(\mathrm{CDCl}_{3}\right) \delta 7.90(\mathrm{~d}, \mathrm{IH}, J=$ $8.0 \mathrm{~Hz}), 7.86(\mathrm{~d}, 1 \mathrm{H}, J=8.4 \mathrm{~Hz}), 7.64-7.49(\mathrm{~m}, 6 \mathrm{H}), 7.47(\mathrm{t}$, $1 \mathrm{H}, J=7.6 \mathrm{~Hz}) ;{ }^{13} \mathrm{C}$ NMR $\left(\mathrm{CDCl}_{3}\right) \delta 148.32,140.98$, $136.58,132.15,129.30,129.20,128.99,127.92,125.65$, $124.94,122.57,114.68,105.86$; IR (KBr) 2209, 1487, 1443, $1354,1262,111 \mathrm{I}, 770,733,694 \mathrm{~cm}^{-1}$.

Ethy] 3-phenylbenzo[b]thiophene-2-carboxylate $7 \mathbf{b}$ : yield $50 \%$; mp 60-61 ${ }^{\circ} \mathrm{C}$ (lit. $\left.{ }^{18} 63-65^{\circ} \mathrm{C}\right){ }^{1} \mathrm{H}$ NMR $\left(\mathrm{CDCl}_{3}\right) \delta 7.89$ $(\mathrm{d}, \mathrm{IH}, J=8.4 \mathrm{~Hz}), 7.56(\mathrm{~d}, 1 \mathrm{H}, J=8.4 \mathrm{~Hz}), 7.53-7.45(\mathrm{~m}$, $4 \mathrm{H}), 7.43-7.39(\mathrm{~m}, 2 \mathrm{H}), 7.36(\mathrm{t}, 1 \mathrm{H}, J=7.6 \mathrm{~Hz}), 4.25(\mathrm{q}, 2 \mathrm{H}$, $J=7.6 \mathrm{~Hz}), 1.2 \mathrm{I}(\mathrm{t}, 3 \mathrm{H}, J=7.6 \mathrm{~Hz}) ;{ }^{13} \mathrm{C} \mathrm{NMR}\left(\mathrm{CDCl}_{3}\right) \delta$ $162.47,143.63,140.27,140.03,134.59,129.57,128.55$, $127.87,126.99,125.15,124.64,122.35,61.20,13.99$ (one carbon is missing due to overlap); IR (KBr) 1723, 1714, $1531,1489,1274,1230,1176,758,736,697 \mathrm{~cm}^{-1}$.

2-Benzoyl-3-phenylbenzo[b]thiophene $7 \mathrm{c}$ : yield $73 \%$; $\mathrm{mp}$ $104-105^{\circ} \mathrm{C}$ (lit. $\left.{ }^{19} 105-106^{\circ} \mathrm{C}\right)$; $^{\prime} \mathrm{H}$ NMR $\left(\mathrm{CDCl}_{3}\right) \delta 7.93$ (d, $1 \mathrm{H}, J=8.0 \mathrm{~Hz}), 7.77(\mathrm{~d}, 1 \mathrm{H}, J=8.0 \mathrm{~Hz}), 7.6 \mathrm{I}(\mathrm{dd}, 2 \mathrm{H}, J=$ $8.0,1.2 \mathrm{~Hz}), 7.49(\mathrm{t}, 1 \mathrm{H}, J=8.0 \mathrm{~Hz}), 7.40(\mathrm{t}, \mathrm{IH}, J=7.6$ $\mathrm{Hz})$, 7.34-7.13 (m, 8H); ${ }^{13} \mathrm{C}$ NMR $\left(\mathrm{CDCl}_{3}\right) \delta 191.45$, $141.27,140.60,139.12,137.61,137.37,134.19,132.23$, $130.18,129.49,128.10,127.85,127.68126 .81,124.95$, $124.92,122.56$; IR (KBr) 1626, 1598, 1517, 1444, 1349, $1289,735,725,703 \mathrm{~cm}^{-1}$.

2-(4-Methoxybenzoyl)-3-phenylbenzo $[b]$ thiophene $7 \mathbf{d}$ : yield $78 \%$; mp 95-97 ${ }^{\circ} \mathrm{C}$ (lit. ${ }^{6} 94-95^{\circ} \mathrm{C}$ ); ${ }^{7} \mathrm{H} \mathrm{NMR}\left(\mathrm{CDCl}_{3}\right) \delta$ $7.92(\mathrm{~d}, 1 \mathrm{H}, J=7.6 \mathrm{~Hz}), 7.78(\mathrm{~d}, 1 \mathrm{H}, J=8.0 \mathrm{~Hz}), 7.66(\mathrm{~d}$, $2 \mathrm{H}, J=8.0 \mathrm{~Hz}), 7.48(\mathrm{t}, 1 \mathrm{H}, J=8.0 \mathrm{~Hz}), 7.40(\mathrm{t}, 1 \mathrm{H}, J=8.0$ $\mathrm{Hz}), 7.32(\mathrm{dd}, 2 \mathrm{H}, J=8.0,1.6 \mathrm{~Hz}), 7.29-7.20(\mathrm{~m}, 3 \mathrm{H}), 6.67$ (d, $2 \mathrm{H}, J=8.0 \mathrm{~Hz}), 3.77(\mathrm{~s}, 3 \mathrm{H}) ;{ }^{13} \mathrm{C} \mathrm{NMR}\left(\mathrm{CDCl}_{3}\right) \delta$ $190.08,163.16,140.38,140.15,139.08,137.72,134.46$, $132.22,130.17,130.14,128.28,127.89,126.51124 .93$, 
$124.73,122.60,113.17,55.37$; IR (KBr) $1629,1592,1283$, $1252,1165,1107,1031,700 \mathrm{~cm}^{-1}$.

2-(4-Methylbenzoyl)-3-phenylbenzo[b]thiophene 7e: yield $80 \%$; mp 86-87 ${ }^{\circ} \mathrm{C}$; 'H NMR $\left(\mathrm{CDCl}_{3}\right) \delta 7.93(\mathrm{~d}, 1 \mathrm{H}, J$ $=8.0 \mathrm{~Hz}), 7.78(\mathrm{~d}, 1 \mathrm{H}, J=8.0 \mathrm{~Hz}), 7.56(\mathrm{~d}, 2 \mathrm{H}, J=8.0 \mathrm{~Hz})$, $7.49(\mathrm{t}, 1 \mathrm{H}, J=8.0 \mathrm{~Hz}), 7.41(\mathrm{t}, 1 \mathrm{H}, J=8.0 \mathrm{~Hz}), 7.31(\mathrm{dd}$, $2 \mathrm{H}, J=8.0,1.6 \mathrm{~Hz}), 7.28-7.20(\mathrm{~m}, 3 \mathrm{H}), 6.98(\mathrm{~d}, 2 \mathrm{H}, J=8.0$ $\mathrm{Hz}), 2.28(\mathrm{~s}, 3 \mathrm{H}) ;{ }^{13} \mathrm{C} \mathrm{NMR}\left(\mathrm{CDCl}_{3}\right) \delta 191.22,143.32$, $140.80,140.52,139.17,137.66,134.82,134.40,130.19$, $129.90,128.54,128.22,127.81,126.68,124.95,124.89$, 122.62, 21.58; IR (KBr) 1624, 1604, 1518, 1484, 1351, $1286,1105,938,778,748,735 \mathrm{~cm}^{-1}$. Anal: Calcd for $\mathrm{C}_{22} \mathrm{H}_{16} \mathrm{OS}$ : C, $80.45 ; \mathrm{H}, 4.91$; S, 9.76. Found: C, 80.59; $\mathrm{H}$, $4.89 ; \mathrm{S}, 9.59$.

\section{References and Notes}

1. Qin, Z; Kastrati, I.; Chandrasena, R. E. P.; Liu, H.; Yao, P.; Petukhov, P. A.; Bolton, J. L.; Thather, G. R. J. J. Med. Chem. $2007,50,2682$.

2. Fedi, V.; Altamura, M.; Calalioto, R.-M.; Giannotti, D.; Giolitti, A.; Giuliani, S.; Guidi, A.; Harmat, N. J. S.; Lecci, A.; Meini, S.; Nannicini, R.; Pasqui, F.; Tramontana, M.; Triolo, A.; Maggi, C. A. J. Med. Chem. 2007, 50,4793.

3. Witter, D. J.; Belvedere, S.; Chen, L.; Secrist, J. P.; Mosley, R. T.; Miller, T. A. Bioorg. Med, Chem. Lett, 2007, 17, 4562.

4. Filzen, G. F.; Bratuon, L.; Cheng, X.-M.; Erasga, N.; Geyer, A.; Lee, C.; Lu, G.; Pulaski, J.; Sorenson, R. J.; Unangsi, P. C.; Trivedi, B. K.; Xu, X. Bioorg. Med. Chem. Lett. 2007, 17, 3630.
5. Romagnoli, R.; Baraldi, P. G; Carrion, M. D.; Cara, C. L.; Preti, D.; Fruttarolo, F.; Pavani, M. G.; Tabrizi, M. A.; Tolomeo, M.; Grimaudo, S.; Cristina, A. D.; Balzarini, J.; Hadfield, J. A.; Brancale, A.; Hamel, E. J. Med. Chem. 2007, 50, 2273.

6. Cullinan, G. J.; Eacho, P. I.; Foxworthy-Mason, P. S.; Schmidt, R. J. EPO 897978 Al, 1999.

7. Scrowston, R. M. Adr. Heterocycl. Chem. 1981, 29, 171.

8. Campaigne, E. Thiophenes and Their Benzo Derivatives: (iii) Symhesis and Applications in Comprehensive Heterooclio Chemistry; Katritzky, A. R., Rees, C. W., Eds.; Pergamon Press: 1984; Vol 4, pp 863-934.

9. Yoshida, S.; Yorimitsu, H.; Oshima, K. Org. Lett, $2007,9,5573$.

10. Willis, M. C.; Taylor, D.; Gillmore, A. T. Tetrahedron 2006, 62, 11513.

11. Kobayashi, K.; Nakamura, D.; Miyamoto, K.; Morikawa, O.; Konishi, H. Bull. Chem. Soc. Jpn. 2007, 80, 1780-1784.

12. Sternbach, L. H.; Lehr, H.; Reeder, E.; Hayes, T.; Steiger, N. $J$. Org. Chem. 1965, 30,2812.

13. Schellenberg, K. A.; Westheimer, F. H. J. Org. Chem. 1965, 30, 1859.

14. Marini, P. J.; Murray, K. S.; West, B. O. J. Chent. Soc. Dalton Trans. 1983, 143.

15. The yield of 2-mercaptobenzophenone oblained from 2-aminobenzophenone via diazolization, reaction with potassium ethyl xanthate, and then hydrolysis of aryl ethyl xanthate was reported to range from 20 to $25 \%$.

16. (a) Overman, L. E.; Smoot, J.; Overman, J. D. Synthesis 1974, 59. (b) Kasmai, H. S.; Mischke, S. G. Synthesis 1989, 763.

17. Emayan, K.; English, R. F.; Koutentis, P. A.; Rees, C. W. J. Chem. Soc, Perkin Trans. I 1997, 3345.

18. Nakamura, Y.; Ukita, T. Org. Lett. 2002, 4, 2317.

19. Oe, K.; Tashiro, M.; Tsuge, O. Bull. Chem. Soc. Jpht 1977, 50,3281. 